\title{
Facile synthesis of poly( $p$-phenylenediamine)/MWCNT nanocomposites and characterization for investigation of structural effects of carbon nanotubes
}

\author{
QUANG LONG PHAM, YUVARAJ HALDORAI, VAN HOA NGUYEN, DIRK TUMA ${ }^{\dagger}$ and \\ JAE-JIN SHIM* \\ School of Display and Chemical Engineering, Yeungnam University, Gyeongsan, Gyeongbuk 712-749, Republic of Korea \\ ${ }^{\dagger}$ BAM Federal Institute of Materials Research and Testing, Richard-Willstätter-Str.11, D-12489 Berlin, Germany
}

MS received 15 October 2010

\begin{abstract}
Poly( $p$-phenylenediamine) (PpPD)/carboxylic acid-functionalized multiwalled carbon nanotubes (cMWCNTs) nanocomposites were prepared by chemical oxidative polymerization using potassium persulfate $\left(\mathrm{K}_{2} \mathrm{~S}_{2} \mathrm{O}_{8}\right)$ as an oxidant. Field-emission scanning electron microscopy (FE-SEM) and field-emission transmission electron microscopy (FE-TEM) showed that a tubular layer of PpPD was coated on the surface of carbon nanotubes with a thickness of 10-20 nm. FT-IR analysis provided an evidence for the formation of nanocomposites. The thermal stability of nanocomposites was improved by addition of $c$-MWCNTs as confirmed by thermogravimetric analysis (TGA). XRD spectra showed that the crystalline nature of PpPD was not affected much by the addition of $c$-MWCNTs. As the content of $c$-MWCNTs was increased, the electrical conductivity of the nanocomposites increased due to the interaction between polymer and nanotubes that enhances electron delocalization.
\end{abstract}

Keywords. $\quad$-Phenylenediamine; multi-walled carbon nanotube; nanocomposite; oxidative polymerization.

\section{Introduction}

The discovery of carbon nanotubes (Iijima 1991) (CNTs) has revolutionarily changed the face of the modern society in various fields of study including physics, materials science and chemistry. Scientists and researchers are attracted to the unique shape, size and remarkable physical performance of the nanotubes that possess a very high tensile strength, high thermal stability, high electrical conductivity in a cylindrical shape with a diameter of around $10 \mathrm{~nm}$ and length of up to $100 \mu \mathrm{m}$. Consequently, nanotubes have found applications in various areas such as electrochemical devices, hydrogen storage, field emission devices, sensor and probes (Baughman et al 2002). The incorporation of CNTs into plastic can potentially provide structural materials with dramatically increased modulus and strength. Dalton et al (2003) reported a spinning method to make super-tough fibres in which the use of CNTs instantly boosted the mechanical strength of poly(vinyl alcohol) (PVA) fibre hundredfold than that of the bare PVA so that it could reach the strength of spider silk. Additionally, the combination of CNTs with conducting polymers enables the production of nanocompo-

\footnotetext{
*Author for correspondence (jjshim@yu.ac.kr)
}

sites with significant improvement in electrical conductivity and thermal stability. Different types of conducting polymers including polyaniline (PANI) (Long et al 2004a), polypyrrole (PPy) (Long et al 2004b), polythiophene (PTh) (Karim et al 2006), and polyacetylene (PA) (Tkachenko et al 2009) were proved to interact well with CNTs as proven by the dramatic increase in the conductivity of polymers. Typically, it was reported that the incorporation of $24.8 \%$ CNTs increased the room temperature conductivity of PANI by hundred times (Long et al 2004a), while 23.1\% incorporation enhanced that of PPy by 30 times (Long et al 2004b).

Recently, investigations on the derivative of PANI, aromatic diamine polymers, are found to be more attractive since they exhibit more novel multifunctionality than PANI and PPy due to one free amino group per repetitive unit on the polymer chains. Thus, these polymers have shown different characteristics from those widely known conducting PANI and PPy in the application of electrocatalysis, electrochromics, sensors, electrode materials, etc (Li et al 2002). Para-phenylenediamine ( $p$ PD), one of the three isomers of phenylenediamine (PD), and its copolymers has been widely studied worldwide. Cataldo (1996) and Li et al (2001) reported polymerization of $p \mathrm{PD}$ by oxidation in aqueous acidic solution with potassium persulfate (KPS) as an oxidant. Poly $(p \mathrm{PD})(\mathrm{P} p \mathrm{PD})$ was believed to have a ladder structure and was somewhat similar to pernigraniline with a specific conductivity of $6.3 \times 10^{-6} \mathrm{~S} / \mathrm{cm}$. In another study, 
PANI was copolymerized with different ratios of phenylenediamine isomers by a chemical oxidative polymerization method (Prokeš et al 1999). Interestingly, poly(aniline-co$p$-phenylenediamine) (poly(Ani-co- $p$ PD)) had conductivity ranging over more than eleven orders of magnitude with the lower end ascribed to the conductivity of $\mathrm{P} p \mathrm{PD}$ that was negligible. More recently, our group (Haldorai et al 2009) synthesized poly(Ani-co- $p$ PD)/multi-walled carbon nanotube nanocomposites by in situ microemulsion polymerization.

The oxidation mechanism of $\mathrm{P} p \mathrm{PD}$ together with its structures has been reported elsewhere (Ichinohe et al 1998; Sestrem et al 2009); however, it is unclear about the final result. The synthesis of $\mathrm{P} p \mathrm{PD} / \mathrm{MWCNTs}$ nanocomposites has not been studied yet. In the present study, we report the preparation of $\mathrm{P} p \mathrm{PD} / c$-MWCNTs nanocomposites by chemical oxidative polymerization. A number of techniques have been used to analyse the nanocomposites such as FT-IR, FE-TEM, FE-SEM, UV-visible, TGA and XRD.

\section{Experimental}

\subsection{Materials}

MWCNTs were purchased from Aldrich ( $>90 \%$ pure) with a diameter of 10-20 nm and length of 0.1-10 $\mu \mathrm{m}$. pPD (>97\%) from Tokyo Chemical Industry and potassium persulfate (KPS) (>99\%) from Aldrich were used as received. Other chemicals were of analytical grade and used without further purification.

\subsection{Oxidation of MWCNTs}

$0.5 \mathrm{~g}$ of MWCNTs was dispersed in $40 \mathrm{ml}$ of concentrated $\mathrm{H}_{2} \mathrm{SO}_{4}$ and $\mathrm{HNO}_{3}$ with a volume ratio of 3:1 using an ultrasonicator for $10 \mathrm{~min}$. The mixture was stirred at $60^{\circ} \mathrm{C}$ for $24 \mathrm{~h}$ and then washed several times with deionized water until the $\mathrm{pH}$ reached 7. The resulting acid-functionalized MWCNTs were filtered with $0.2 \mu \mathrm{m}$ PTFE membrane filter and dried in a vacuum at $70^{\circ} \mathrm{C}$ for $24 \mathrm{~h}$. This treatment produced carboxylic acid groups $(-\mathrm{COOH})$ on the surfaces of MWCNTs at their defects and shortened the length of the tubes.

\subsection{Preparation of PpPD}

In a typical experiment, $0.015 \mathrm{~mol} p \mathrm{PD}$ was added into a $200 \mathrm{ml}$ flask containing $100 \mathrm{ml}$ of acidic aqueous solution $(\mathrm{HCl}, 0 \cdot 1 \mathrm{M})$. Then, the mixture was pre-cooled and stirred constantly in an ice bath to make a homogenous solution. The oxidant solution was prepared by dissolving $0.015 \mathrm{~mol}$ KPS in $50 \mathrm{ml}$ of $0 \cdot 1 \mathrm{M} \mathrm{HCl}$. After that, the oxidant solution was added dropwise to the monomer solution for $30 \mathrm{~min}$ to initiate the polymerization. While stirring, the reaction system was kept for $24 \mathrm{~h}$ at room temperature to assure the completion of reaction. Then, it was terminated by pouring acetone into the reaction mixture. The product was washed completely with deionized water to remove impurities such as unreacted monomer, oxidant and $\mathrm{HCl}$. It was then washed with acetone, filtered and dried under a vacuum at $60^{\circ} \mathrm{C}$ for $24 \mathrm{~h}$.

\subsection{Preparation of PpPD/c-MWCNTs nanocomposites}

Each of 2.5 and $5 \mathrm{wt} \% c$-MWCNTs (based on the weight of $p \mathrm{PD}$ ) was dispersed in the solution of $0.015 \mathrm{~mol} p \mathrm{PD}$ in $100 \mathrm{ml}$ of $0.1 \mathrm{M} \mathrm{HCl}$ by ultrasonication for $10-15 \mathrm{~min}$. Then, the solution of $0.015 \mathrm{~mol} \mathrm{KPS}$ in $50 \mathrm{ml}$ of $0.1 \mathrm{M}$ $\mathrm{HCl}$ was added drop by drop into the previous solution which was stirred constantly in an ice bath in a period of $30 \mathrm{~min}$ to initiate the polymerization. The reaction was kept for $24 \mathrm{~h}$. Acetone was then poured into the reaction mixture to stop polymerization and to precipitate the $\mathrm{P} p \mathrm{PD} / c$ MWCNTs nanocomposite. The purification and drying procedures were the same as those for the synthesis of the bare polymer.

\subsection{Characterization}

The morphology of nanocomposites was determined by FESEM (Hitachi, S-4100). Microstructures of the samples were examined by FE-TEM (FEI, Tecnai G2 F20). TGA was performed on a thermal analyser (TA Instruments, SDT Q600) from $40-800^{\circ} \mathrm{C}$ at a heating rate of $10^{\circ} \mathrm{C} / \mathrm{min}$ under nitrogen atmosphere. For confirmation of crystallinities and structures of $c$-MWCNTs, bare P $p$ PD and nanocomposites, X-ray diffraction (XRD) data were collected with a diffractometer (Panalytical, X'Pert Pro MPD) using $\mathrm{Cu}-\mathrm{K} \alpha$ radiation sources at an operating voltage of $40 \mathrm{kV}$. Structural effects of nanocomposites were characterized by FT-IR spectrometer (Bio-Rad, Excalibur FTS 3000) and UV-Visible spectrophotometer (Shimadzu, UV-160A). Room temperature conductivity was measured by the Hall Effect Measurement System (Ecopia, HMS-5000) using the Van der Pauw fourprobe method.

\section{Results and discussion}

\subsection{Acid-functionalized MWCNTs}

Carbon nanotubes functionalized with carboxylic acid groups were confirmed by FT-IR as shown in figure 1 . Two bands at around 3350 and $1200 \mathrm{~cm}^{-1}$ are assigned to the $-\mathrm{OH}$ groups on the surface of pristine MWCNTs (Haldorai et al 2009), which might originate from ambient moisture bound to the MWCNTs or be generated during purification of the raw material (Ramanathan et al 2005). In the acid-functionalized MWCNTs spectrum, the peak near $1560 \mathrm{~cm}^{-1}$ corresponds to the infrared-active phonon mode of the nanotubes (Jeevananda et al 2008) and the peaks at 


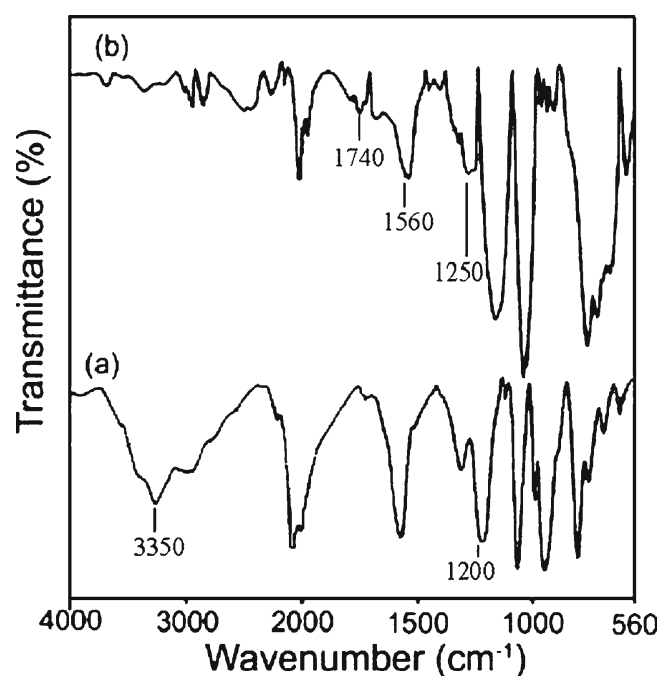

Figure 1. FT-IR spectra of (a) pristine MWCNTs and (b) $c$-MWCNTs.

1740 and $1250 \mathrm{~cm}^{-1}$ apparently correspond to the stretching mode of the carboxylic acid groups (Mawhinney et al 2000). These observations are clear indications of $-\mathrm{COOH}$ groups on the surface of nanotubes.

The difference in the morphology between pristine MWCNTs and $c$-MWCNTs was confirmed by FE-SEM as shown in figure 2. Pristine MWCNTs which have smooth surfaces appeared as large bundles with lengths in the order of micron and diameters around 10-20 nm. After acid treatment, the $c$-MWCNTs were disentangled and rough, and their lengths were slightly reduced by both the oxidation and ultrasonic treatment.

\subsection{Morphology and structure of bare polymer and nanocomposites}

Scheme 1 interprets the formation of $\mathrm{P} p \mathrm{PD} / c$-MWCNTs nanocomposite. There are three main steps represented by three arrows. The first step is the surface modification of MWCNTs which introduces $\mathrm{COOH}$ groups as well as changes the surface morphology of the nanotubes as confirmed by FT-IR and FE-SEM. The second step is the addition of as-prepared $c$-MWCNTs into the acidic solution of $p \mathrm{PD}$ monomer followed by ultrasonication. Interactions between monomer and the surface of $c$-MWCNTs are depicted in the enlarged picture. Basically, there are two main interactions: one is the H-bonding interaction between the proton in the carboxylic group of $c$-MWCNT and $\mathrm{N}$ atoms of monomer, another one is the $\pi-\pi^{*}$ interaction between benzenoid ring of $p \mathrm{PD}$ and the $\pi$-system of CNT. Such interactions pledge the strong encapsulation of $c$-MWCNTs by $\mathrm{P} p \mathrm{PD}$ which is formed in polymerization step (the third step). In this last step, polymer is formed by
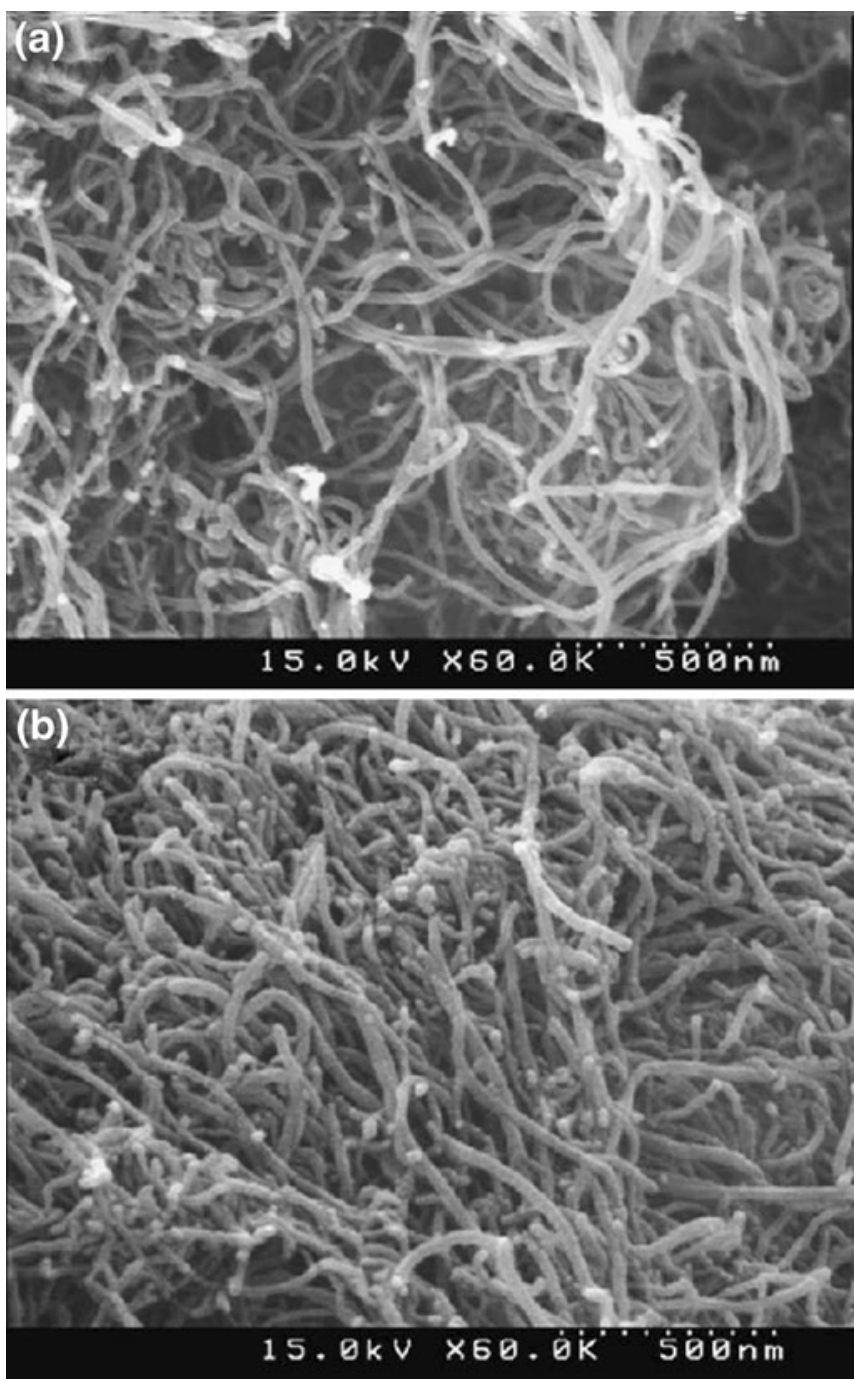

Figure 2. FE-SEM micrographs of (a) pristine MWCNTs and (b) $c$-MWCNTs.

chemical oxidative polymerization of $p \mathrm{PD}$ with an aid of an oxidant, $\mathrm{K}_{2} \mathrm{~S}_{2} \mathrm{O}_{8}$ and the growth of polymer takes place both on the surface of $c$-MWCNTs and in the continuous phase. However, the polymerization reaction is faster on the surface of $c$-MWCNTs in comparison with the continuous phase due to lower activation energy (based on the principle of heterogeneous catalyst).

FE-SEM micrographs of the bare $\mathrm{P} p \mathrm{PD}$, and nanocomposite with $2.5 \% c$-MWCNTs are shown in figure 3 . The bare polymer cropped up with non-descriptive shapes, while in the case of $\mathrm{P} p \mathrm{PD} / c$-MWCNTs nanocomposite, a tubular polymer layer was coated on the surface of carbon nanotubes, thus making the diameters of the composites larger than that of $c$-MWCNTs as reflected in figure $2 \mathrm{~b}$. In order to confirm the coating of polymer on the surface of carbon nanotubes, FE-TEM image of the composite with $2.5 \% c$-MWCNTs 


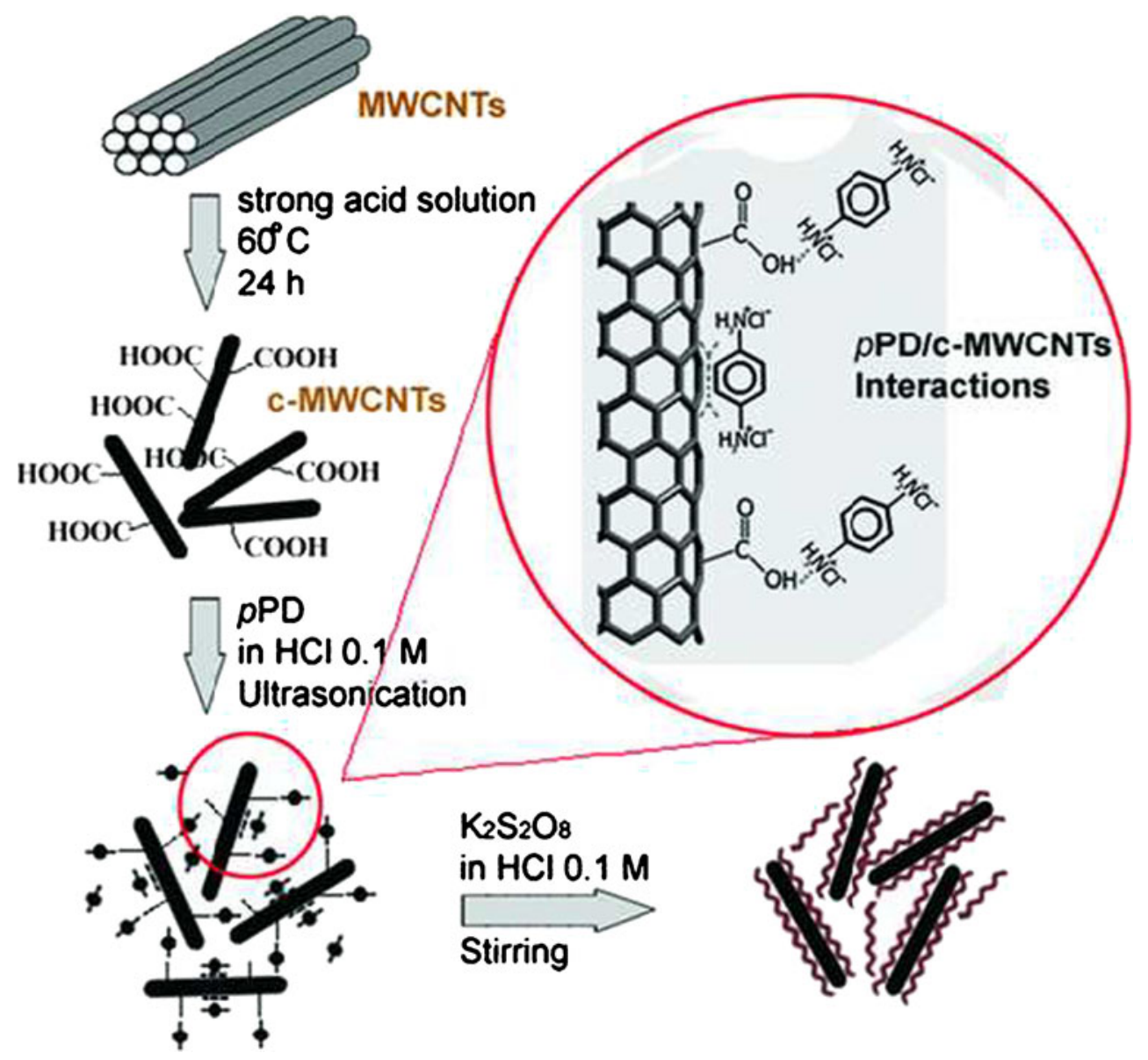

Scheme 1. A schematic illustration of the formation of $\mathrm{P} p \mathrm{PD} / c$-MWCNTs nanocomposite.

was taken. A core-shell structure was clearly observed in its expanded view (figure 3d). The core is a nanotube with a diameter of $10-20 \mathrm{~nm}$, whereas the shell is made up of the polymer with a thickness of 10-20 nm. As shown in figure $3 \mathrm{c}$, the polymer layer was uniformly grown on the surface of nanotubes to form the tubular nanocomposite. Formation of the polymer coating on the surface of CNTs can be explained by the absorption of $p \mathrm{PD}$ monomer onto the surface of CNTs during polymerization (Jeevananda et al 2008).

\section{$3.3 U$ U-Vis spectra}

UV-Visible spectra of the bare polymer and nanocomposite with 5\% c-MWCNTs in N-methyl-2-pyrrolidone (NMP) are shown in figure 4. Both samples showed similar curve patterns. A shoulder band at around $270 \mathrm{~nm}$ and a strong absorption band at $305 \mathrm{~nm}$ are assigned to the $\pi-\pi^{*}$ transition of the benzenoid rings of the polymer (Haldorai et al 2009). The broad band centred at $\sim 430 \mathrm{~nm}$ is attributable to the polaron $-\pi$ transition on $\mathrm{P} p \mathrm{PD}$ chain, typically, in some places it resembles the emeraldine salt structure of polyani- line (PANI-ES) (Wu et al 2005; Sestrem et al 2009). There is a slight difference in the spectra of $\mathrm{P} p \mathrm{PD} / c$-MWCNT composite from those of the bare polymer. By comparing the relative heights for the peaks at $305 \mathrm{~nm}$ and $430 \mathrm{~nm}$, we can see that the band at $430 \mathrm{~nm}$ in the nanocomposite showed a higher absorption than that in the bare polymer. This result suggests that there is an interaction between the quinoid rings of $\mathrm{P} p \mathrm{PD}$ and $c$-MWCNTs that enhances the formation of the polaron $-\pi$ transition of the polymer chain.

\section{$3.4 \quad F T-I R$ spectra}

FT-IR spectra of the bare polymer and nanocomposites with $2.5 \%$ and $5 \% c$-MWCNTs are shown in figure 5. A broad peak centred at around $3450 \mathrm{~cm}^{-1}$ originates from the $\mathrm{N}-\mathrm{H}$ stretching vibration of secondary amine group in the polymer chain. The peaks at 1602 and $1493 \mathrm{~cm}^{-1}$ are assigned to the $\mathrm{C}=\mathrm{N}$ and $\mathrm{C}=\mathrm{C}$ stretching vibrations in quinoid and benzenoid rings, respectively ( $\mathrm{Li}$ et al 2002). The indication of the imine $\mathrm{C}-\mathrm{N}$ absorption showed a small peak at about $1300 \mathrm{~cm}^{-1}$, and the peak at $830 \mathrm{~cm}^{-1}$ can be ascribed to the out-of-plane bending vibration of benzene ring 

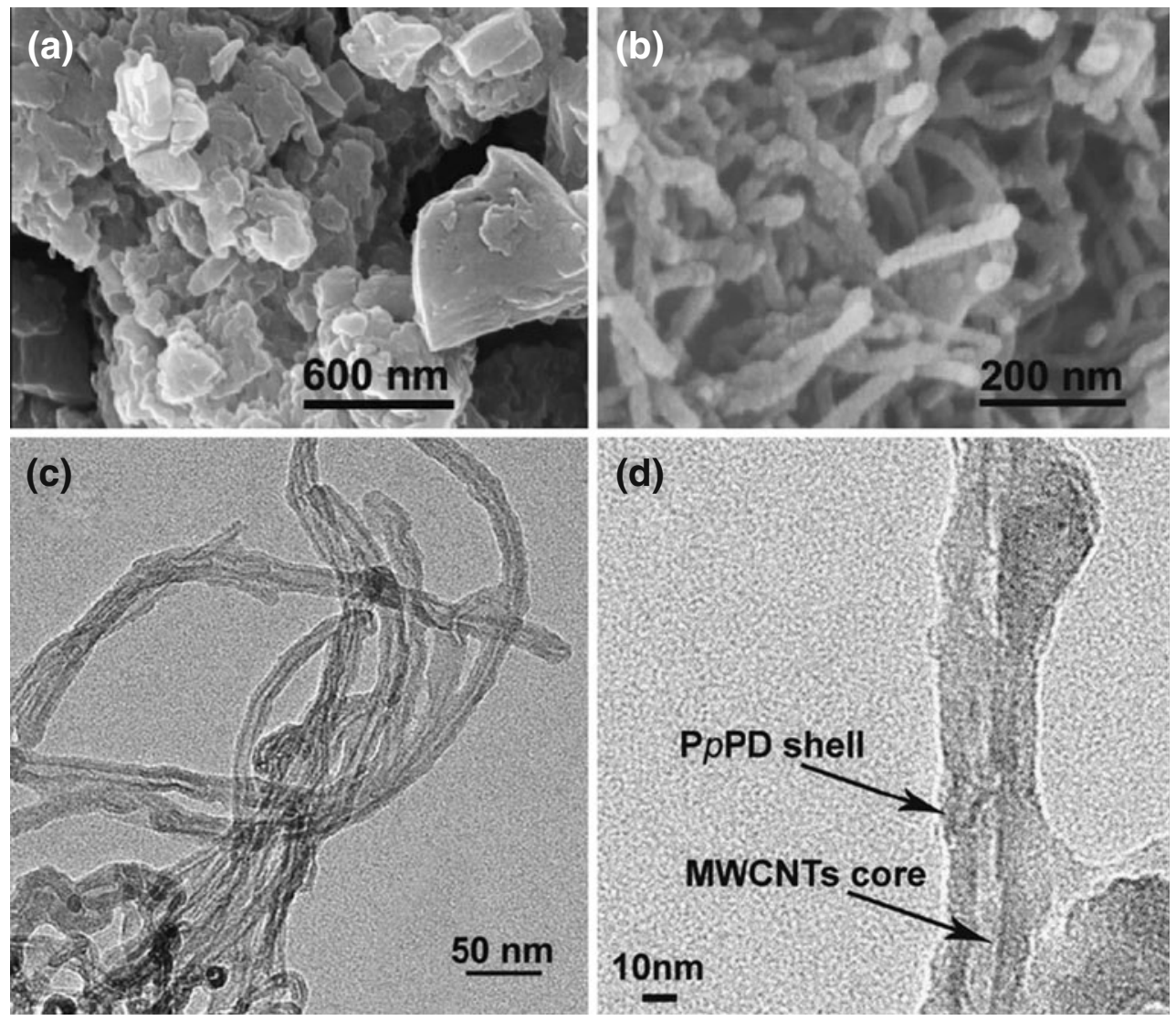

Figure 3. FE-SEM micrographs of (a) bare polymer and (b) nanocomposite with $2 \cdot 5 \% c$-MWCNTs. FE-TEM micrographs of (c) nanocomposite with $2.5 \% c$-MWCNTs and (d) its expanded view.

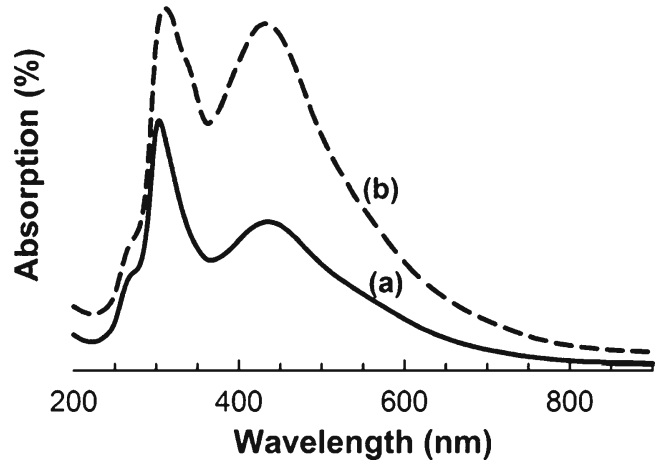

Figure 4. UV-visible spectra of (a) bare polymer and (b) nanocomposite with 5\% c-MWCNTs.

(Haldorai et al 2009). The strong band at around $1132 \mathrm{~cm}^{-1}$ was supposed to be "electron-like band" which is considered to be a measure of delocalization (Cataldo 1996). The change in the intensity ratio of the quinoid and benzenoid peaks was observed between the bare polymer and composites. This result may be due to the discrepancy in the polymer structures of those polymerized in the continuous phase and those growing on the surface of carbon nanotubes. In the latter case, less benzenoid units were formed, as evidenced by the reduction in the relative strength of IR signals at $1602 \mathrm{~cm}^{-1}$ and $1493 \mathrm{~cm}^{-1}$. Similar results were observed by others also (Cataldo 1996).

\subsection{XRD spectra}

In order to confirm the influence of carbon nanotubes on the crystal structure of P $p$ PD, XRD spectra of $c$-MWCNTs, bare polymer, and nanocomposites of $2.5 \% \mathrm{c}$-MWCNTs and $5 \% c$-MWCNTs are shown in figure 6. Diffraction pattern of $c$-MWCNTs is characterized by a strong peak at $2 \theta=$ $25.9^{\circ}$ which is ascribed to the graphite-like structure (Wu et al 2005). In the spectrum of bare polymer, there are several peaks that represent the crystalline nature of the polymer: a low-intensity broad peak at $14^{\circ}$, a high-intensity sharp peak at around $16 \cdot 3^{\circ}$, a broad peak at $18 \cdot 3^{\circ}$, and a sharp peak at $19 \cdot 1^{\circ}$. A large, broad bump of a low-intensity peak centred at $25^{\circ}$ is attributed to the amorphous portion of P $p$ PD synthesized by oxidative polymerization that fairly correlates with XRD spectrum of P $p$ PD reported by Li et al (2001). 


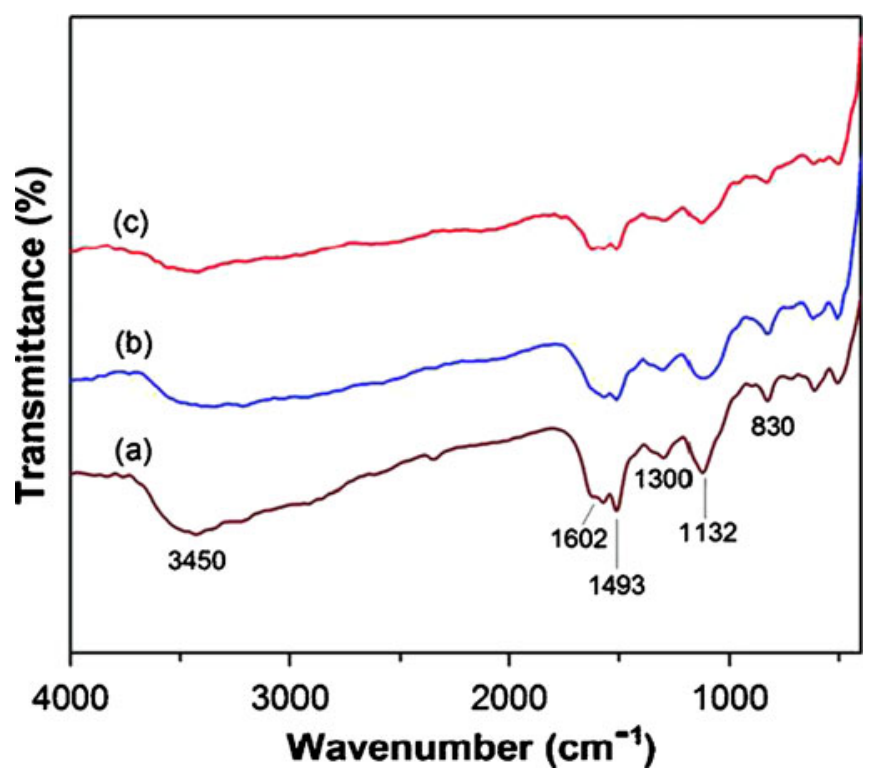

Figure 5. FT-IR spectra of (a) bare polymer, (b) nanocomposite with $2.5 \% c$-MWCNTs and (c) nanocomposite with $5 \%$ $c$-MWCNTs.

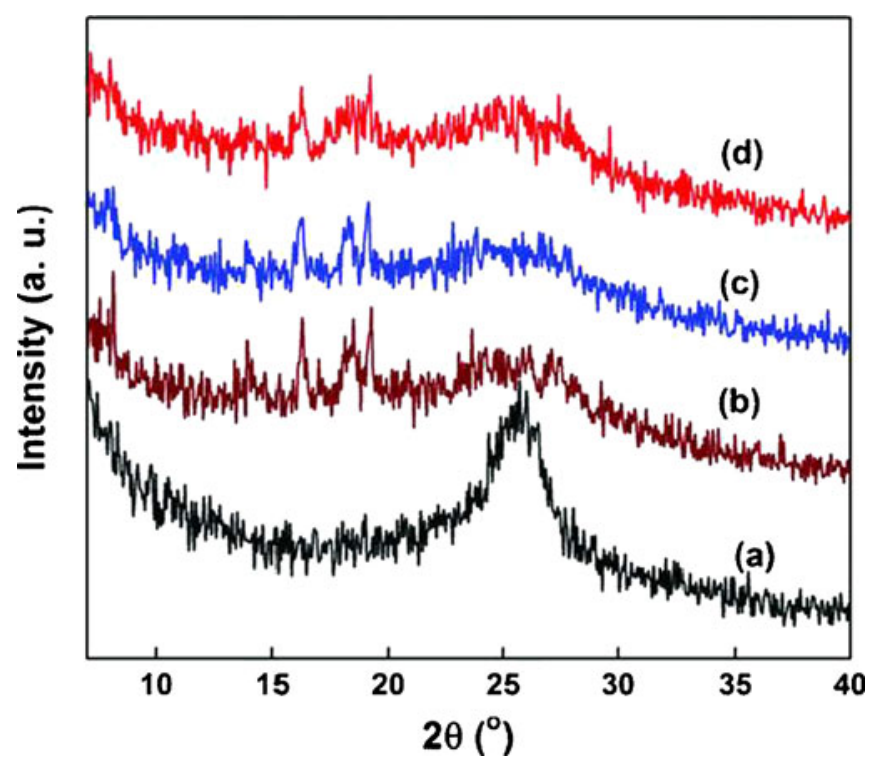

Figure 6. XRD patterns of (a) $c$-MWCNTs, (b) bare polymer, (c) nanocomposite with 2.5\% c-MWCNTs and (d) nanocomposite with $5 \% c$-MWCNTs.

The addition of $c$-MWCNTs into the polymer matrix did not cause a discernible change in the lattice structure of $\mathrm{P} p \mathrm{PD}$ as can be seen in figure 6 . There was only a small reduction in the sharpness of the crystalline peaks, as CNTs were present in the polymer matrix and it slightly changed the crystal nature of the bare polymer.

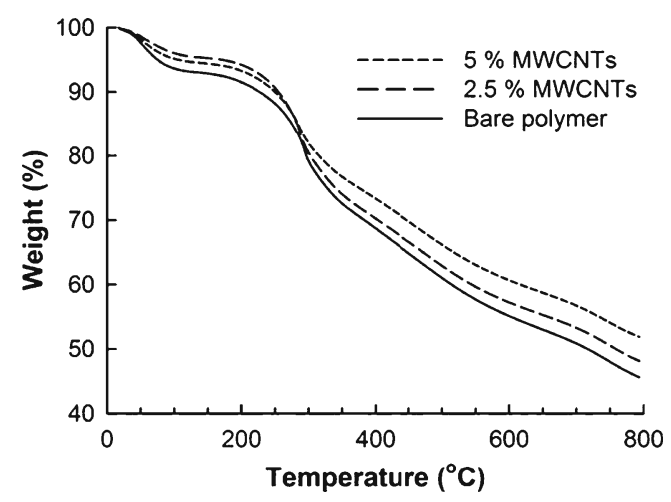

Figure 7. TGA curves of the bare polymer and nanocomposites in nitrogen.

\subsection{Thermal stability}

Thermal stability of bare polymer and nanocomposites is shown in figure 7 . The shapes of those curves are similar to each other, as they reflect two main stages of weight loss. The first weight loss step between 50 and $140^{\circ} \mathrm{C}$ is due to the loss of moisture, vaporization of solvent, and residual $\mathrm{HCl}$ absorbed by the polymer. The second step starting from $170^{\circ} \mathrm{C}$ is attributed to the decomposition of polymer. We can see that the bare polymer decomposed at a higher rate than polymer in the nanocomposites. The extent of decomposition declined as more $c$-MWCNTs were added, as evidenced by less weight loss in the $5 \mathrm{wt} \%$ sample in comparison with the $2.5 \mathrm{wt} \%$ one. This suggests that the addition of $c$-MWCNTs improved the thermal stability of polymer that was correlated well with other studies (Jeevananda et al 2008; Haldorai et al 2009).

\subsection{Conductivity}

Conductivity of bare polymer and nanocomposites was measured using a four-point probe method at room temperature. The results are given in table 1 . According to the previous studies (Cataldo 1996; Prokeš et al 1999; Lakard et al 2003), conductivity of neat $\mathrm{P} p \mathrm{PD}$ is nearly negligible, so it is not surprising that we could not get any data for this sample because its conductivity was below the lower limitation of the instrument. This indicates that the bare polymer is almost

Table 1. Conductivities of bare polymer and nanocomposites measured at 300K. (Data shown here represent the mean measurement values of at least 3 different positions on the surfaces of the samples).

\begin{tabular}{lc} 
Samples & Conductivity (S/cm) \\
\hline Bare P PPD & N/A \\
Nanocomposites with 2.5\% c-MWCNTs & $\sim 2 \times 10^{-6}$ \\
Nanocomposites with 5\% c-MWCNTs & $\sim 8 \times 10^{-3}$ \\
\hline
\end{tabular}


an insulator. In contrast, the nanocomposites showed better conductivity and the conductivity increased with increasing nanotube content. Interestingly, with increase in $c$-MWCNTs content from $2.5 \%$ to $5 \%$, the conductivity of the nanocomposite increased by three orders of magnitude. This improvement is due to the $\pi-\pi^{*}$ interaction between the surfaces of $c$-MWCNTs and the quinoid ring of P $p$ PD (Haldorai et al 2009), which effectively improved the degree of electron delocalization between the two components, as confirmed by FT-IR and UV-Vis spectra.

\section{Conclusions}

We have successfully synthesized nanocomposites consisting of P $p$ PD and acid-functionalized MWCNTs using chemical oxidative polymerization. Morphological studies by FESEM and FE-TEM revealed the core-shell structure of nanocomposite with nanotube as the core and a coating layer of $\mathrm{P} p \mathrm{PD}$ as the shell with a thickness of $10-20 \mathrm{~nm}$. The addition of $c$-MWCNTs into the polymer improved both thermal stability and electrical conductivity, but did not influence the crystalline nature of the bare polymer to any great extent, as confirmed by XRD. The bare polymer was almost an insulator; however, the nanocomposites showed better conductivity, ranging from $2 \times 10^{-6}$ to $8 \times 10^{-3} \mathrm{~S} / \mathrm{cm}$. The improved conductivity can apparently be understood from the interaction between $c$-MWCNTs and the polymer chains.

\section{Acknowledgement}

This work was supported by the Korea Research Foundation Grant funded by the Korean Government (KRF-20100016445).

\section{References}

Baughman R H, Zakhidov A A and de Heer W A D 2002 Science 297787

Cataldo F 1996 Eur. Polym. J. 3243

Dalton A B, Collins S, Muñoz E, Razal J M, Von Howard E, Ferraris J P, Coleman J N, Kim B G and Baughman R H 2003 Nature 423 703

Haldorai Y, Lyoo W S and Shim J J 2009 Colloid Polym. Sci. 287 1273

Ichinohe D, Saitoh N and Kise H 1998 Macromol. Chem. Phys. 199 1241

Iijima S 1991 Nature 35456

Jeevananda T, Siddaramaiah, Kim N H, Heo S B and Lee J H 2008 Polym. Adv. Technol. 191754

Karim M R, Lee C J and Lee M S 2006 J. Polym. Sci. Part A: Polym. Chem. 445283

Lakard B, Herlem G, Lakard S and Fahys B 2003 J. Mol. Struct. (Theochem.) 638177

Li X G, Huang M R, Chen R F, Jin Y and Yang Y L $2001 \mathrm{~J}$. Appl. Polym. Sci. 813107

Li X G, Huang M R and Duan W 2002 Chem. Rev. 1022925

Long Y Z, Chen Z J, Zhang X T, Zhang J and Liu Z F 2004a Appl. Phys. Lett. 851796

Long Y Z, Chen Z J, Zhang X T, Zhang J and Liu Z F 2004b J. Phys. D: Appl. Phys. 371965

Mawhinney D B, Naumenko V, Kuznetsova A, Yates Jr. J T, Liu J and Smalley R E 2000 J. Am. Chem. Soc. 1222383

Prokeš J, Stejskal J, Křivka I and Tobolková E 1999 Synth. Met. 102 1205

Ramanathan T, Fisher F T, Ruoff R S and Brinson L C 2005 Chem. Mater. 171290

Sestrem R H, Ferreira D C, Landers R, Temperini M L A and do Nascimento G M D 2009 Polymer 506043

Tkachenko L I, Efimov O N, Anoshkin I V, Kulova T L, Roshchupkina O S, Shul'ga Yu M and Petrova N K 2009 Russ. J. Electrochem. 45296

Wu T M, Lin Y W and Liao C S 2005 Carbon 43734 